This item was submitted to Loughborough's Research Repository by the author.

Items in Figshare are protected by copyright, with all rights reserved, unless otherwise indicated.

\title{
Information about medicines for patients in europe: To impede or to
} empower?

\section{PLEASE CITE THE PUBLISHED VERSION}

https://doi.org/10.1007/978-3-319-07635-5_14

\section{PUBLISHER}

Springer

VERSION

AM (Accepted Manuscript)

\section{PUBLISHER STATEMENT}

The final authenticated version is available online at https://doi.org/10.1007/978-3-319-07635-5_14.

LICENCE

CC BY-NC-ND 4.0

\section{REPOSITORY RECORD}

Van-Der-Waarde, Karel. 2020. "Information About Medicines for Patients in Europe: To Impede or to Empower?". Loughborough University. https://hdl.handle.net/2134/12957293.v1. 


\title{
Information about medicines for patients in Europe: to impede or to empower?
}

\author{
Karel van der Waarde \\ Graphic Design - Research, Solariumlaan 15, 1982 Elewijt, Belgium \\ waarde@glo.be
}

\begin{abstract}
Information about medicines in Europe does not really fulfil its potential. For patients, it is often very hard to understand and to apply information in a specific situation. For the pharmaceutical industry, it is hard to develop (writing-designing-testing) due to strict regulations. And for the Regulatory authorities, the current situation is hard to control and check. One of the main causes is that legal-, economic-, and health-criteria are simultaneously applied to information about medicines. However, these three criteria are fundamentally different, and have proved to be unbridgeable in the last 20 years. In order to provide patients with usable information, it seems essential to develop a legal system that is not based on standardization of processes and results, but in stead is based on required performances in context.
\end{abstract}

Keywords: Patient information, medicine packaging, labelling, information design.

\section{Situation: patients, industry, and legislation}

Patients in Europe receive a substantial amount of information about their medicines. This information is supplied on packaging, in package leaflets, and on inner packaging' such as blister packs, tubes, or bottles. Pharmacists can add handwritten instructions and additional printed labels, and sometimes provide auxiliary letters and brochures. Especially when patients use more than one medicine, this uncoordinated information supply frequently exceeds the processing capacity of patients. The overload of visual information leads to unwanted side effects like a reduced confidence in the pharmaceutical industry ("they are just covering their backs" - see figure 1), reduced confidence in pharmacists and doctors ("they just try to give you the cheapest ones" - see figure 2) and difficult risk-benefit analyses ("it might help, but it could get worse too?" - see figure 3 ).

Some of the information supply is heavily regulated and controlled within a complex framework of regulations and guidelines. Other - unregulated - information is added at different stages, and this has sedimented gradually over the years into a structure that is hard to handle, difficult to read, and very hard to understand or apply. The breaking point has been reached where regulations prevent progress and hamper the supply of relevant and useful information to patients. 


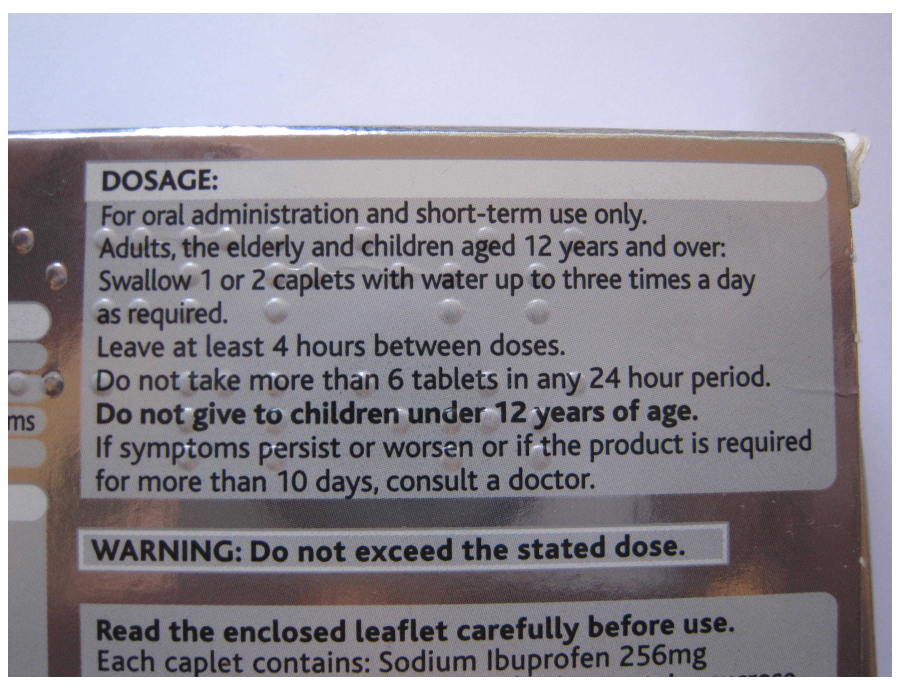

Fig. 1. Example of a detail of an outer pack. The number of warnings and the position of the warnings make it difficult for patients to follow the advice. The interpretation of the combination of 'Do not take more than 6 tablets in any 24 hour period.' and 'WARNING: Do not exceed the stated dose.' requires some mental agility. It is unclear why both are mentioned. The readability of the text is further severely reduced by the use of a highly reflective silver background, a small type size, and braille dots.

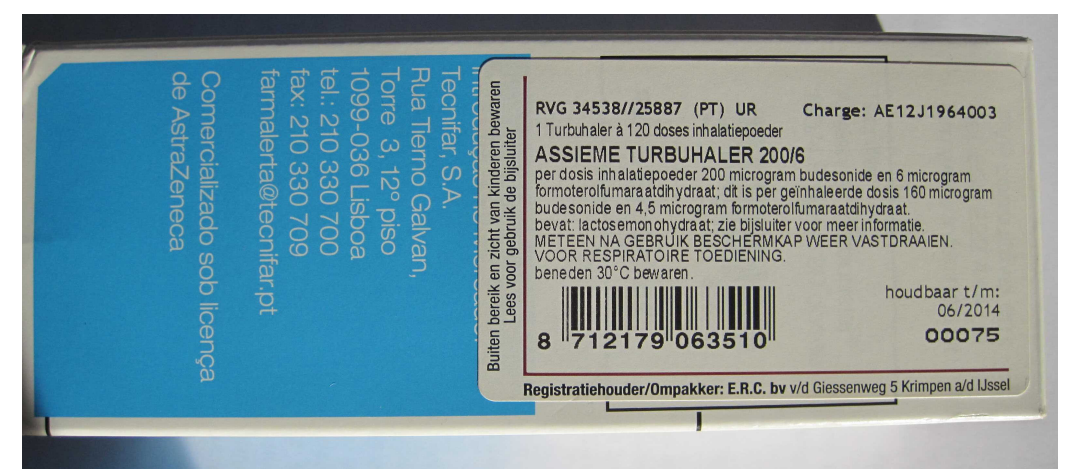

Fig. 2. Example of additional labelling. In order to maximize income, it is possible to buy medicines across Europe (parallel import). The consequence is for example that Dutch patients might receive a Portuguese package with texts in Portuguese. This is remedied by an additional sticker on the outer packing with Dutch texts. Unfortunately, this makes it very unclear who is responsible for the supply of this information. It could be the importer, the pharmacist, the Portuguese manufacturer, or the actual licence holder. 
NSAIDs provide relief by changing the body's response

to pain, swelling, and high temperature. Nurofen

Express $256 \mathrm{mg}$ Caplets are used for the relief of fever

and mild to moderate pain, such as :

- Headaches and migraine pain

- Neuralgia, Backache, period pain, rheumatic and

muscular pain

- Cold and Flu symptoms, sore throat

Tell your doctor if you experience:

- fever, flu-like symptoms, sore throat, mouth ulcers,

headache, a stiff neck, vomiting, unexplained

bleeding and bruising, severe exhaustion.

- indigestion, stomach or abdominal pain, constipation, diarrhoea, flatulence or if you feel sick, chest pain or fast, irregular heart beat.

- liver and kidney problems associated with swelling in your arms and legs.

Fig. 3. Package leaflet details. Top: detail of front. Bottom: Detail of the back of the same leaflet. A medicine that is used 'for the relief of fever and mild to moderate pain' (top image) might cause 'fever' and 'headaches' as side effects (bottom image). Technically, this information is likely to be correct, but it is difficult for a patient to distinguish between the 'headache before taking the tablets', and the 'headache that is caused by the tablets'.

All three examples are in conflict with the intention of the European legislation. A European Directive formulates this intention as 'information supplied to users should provide a high degree of consumer protection, in order that medicinal products may be used correctly on the basis of full and comprehensible information.'[1]. The examples are also in conflict with the actual articles in the EU-legislation which state that 'The package leaflet must be written and designed to be clear and understandable, enabling the users to act appropriately, when necessary with the help of health professionals.' [2] Most information about medicines for patients in Europe fails these criteria.

Of course this has been known for a long time, and in December 2010 a Directive mentions that 'improvements' are required. Directive 2004/84 states: 'By 1 January 2013, the Commission shall present to the European Parliament and the Council an assessment report on current shortcomings in the summary of product characteristics and the package leaflet and how they could be improved in order to better meet the needs of patients and healthcare professionals.' [3]

At the time of writing (February 2014), this report has not been published yet. The delay in the publication might be an indication of the unforseen difficulties to deal with a real 'wicked problem'. 


\subsection{Questions}

It is clear that the information supply to patients does not always 'enable patients to take medicines correctly'. Investigations over the last years [4] point to the necessity to reframe the situation towards 'the activities of people'. It is clear that an analysis of the fundamental activities and expectations of patients is required to find out what patients really believe and need when they interpret information about their medicines. But there are two other main stakeholders. The pharmaceutical industry and the Regulatory authorities are directly involved too. Their activities and expectations, albeit very different from patients, need to be considered too.

Any legislation should therefore take account of the activities of at least three groups:

a. legislation must enable regulators to check and control. The current legislation, guidelines, and templates do not provide enough grip on the matter. Many issues remain unresolved and need to be dealt with on 'a case by case basis'. This reduces the confidence in the regulatory system because it has lead to insonsistency in decisions.

b. legislation about information about medicines must enable people to act appropriately. The aim of the legal framework is to provide patients with relevant and useful information. The contents (figure 3) and design (figure 2) leaves much to be desired, although these examples have been approved and are therefore considered to be 'appropriate'.

c. legislation must support the pharmaceutical industry to develop information, and - if required - develop innovative artefacts. The current legal framework stifles and frustrates both these activities. Although it is absolutely essential to indicate a 'minimally acceptable level', it should also be possible to excel and make progress.

And this combination of requirements of these three groups is probably the main reason that it is so difficult to develop a report about the current shortcomings. Again, this is not new. All three groups have noticed this and discussed their views. It becomes now obvious that there must be some more fundamental reasons why intervention ambitions do not reach the different environments. Two questions should be answered:

- What are the reasons that progress does not occur? Can these reasons be overcome? (section 2).

- Could a performance based design process based on thorough user experience research provide an alternative? (section 3 )

\subsection{Answering the questions?}

Different kinds of testing, such as usability tests, readability tests, diagnostic tests and performance tests have been performed on different types of medicines and different groups of patients. Interviews with the pharmaceutical industry have been conducted at different stages. One group of stakeholders that has only be informally interviewed are the people who work for the national and European regulatory authorities. The 
text below highlights some patterns from the results of these tests and interviews. Although it would be useful to analyse these results in a more formal manner, it is likely to be too timeconsuming. Changes in regulatory framework occur very often, and it is likely that a thorough analysis would become irrelevant well before it is published.

\section{Patterns preventing change}

The three main assumptions that hamper progress are related to the differences between medicines, visual information development processes, and the lack of an acceptable quality control process.

\subsection{Assumption 1: information about different medicines can follow identical structures.}

The European Medicines Agency (EMA) has developed a template that must be followed [5]. The template - currently version 9 - includes information on packaging and package leaflet and is available in 24 languages. All EU-packaging and EUpackage leaflets are now based on this template. There is a range of guidelines and advice that is related to the the interpretation of this template.

However, different tests show that there are substantial and relevant differences between patients. Information about medicines for health care professionals, caregivers, adults, and for the elderly might need to vary, as well as information for experienced and newly diagnosed patients. At the moment all these differences are ignored by the template: every patient receives information in the same rigid structure.

There are also fundamental differences between medicines. Patients clearly indicate that medicines for short term use, such as antibiotics, painkillers, or cough-syrups, are perceived differently from long term medicines for chronic conditions like high blood pressure or high cholesterol levels. Medicines to treat diabetes and HIV-infections are seen as yet another category. And the location, such as hospitals or at home are seen as different too. The EMA-template does not cater sufficiently for these differences.

Unfortunately, the legislation, regulations, and templates start from an assumption that 'all medicines are the same' and that it is possible to standardize all information. A comparison of the results of 'readability tests' of medicines information suggests that this assumption is incorrect. 
2.2 Assumption 2: writing, designing, and testing are simple rule bound practices

The use of a standardized template [5], rigid design guidelines [6], and standardized tests [6] is very hard to apply in practice. The assumption that underlies the legislation is that 'writing, designing, testing' are three consecutive and separate activities. The pharmaceutical industry develop a written texts using the template as a starting point and adding texts in between standardized statements in a unalterable structure. This text is formatted and designed according to some suggestions in the Readability guideline in combination with corporate guidelines. The design boundaries are further set by production requirements which dictate the maximum dimensions of the packaging and the package leaflet. According to EU-legislation, it is only necessary to test the package leaflet in one language. The packaging and information in additional languages is very rarely tested.

This disjointed approach, in which there is hardly a relation between the text, the visual design, and the testing requirements results in visual information that is not really satisfying anyone.

The pharmaceutical industry keeps therefor asking three questions:

- Are we writing the correct information?

- Are we designing the correct artefacts?

- Are we testing the correct actions?

For most medicines, none of these three questions can be answered positively.

From a regulatory point of view, this disjointed approach causes severe problems too. Although it is possible to check if a text follows a template, it is not possible to check if a text really is suitable for patients. The results of the readability tests are always positive and will show that patients can find and understand the main points. Howeveer, the test reports will rarely indicate any issues that patients had with the standardised texts in the template. This is because a change in the standardised template texts might not be approved and this could lead to costly delays. It is very clear that the regulatory authorities take great care to make sure that information is as good as possible. However, there is a lack of suitable tools and guidelines that would help to check the quality of a text, to assess a visual design, and to evaluate test results.

Both the pharmaceutical industry as well as the regulatory authorities struggle with the current three-step process of writing, designing, and testing. The assumption that these are easily done and easily controlled proved to be incorrect.

\subsection{Assumption 3: A digital submission and control system is not required.}

In the last 20 years, the different European regulatory authorities and the pharmaceutical industry have dealt with enormous numbers of packaging, labelling, and leaflets. All packaging and leaflets have been reconsidered, modified, and are still 
in the process of change to conform to continuously updated legislation. Unfortunately, this process has not been set up as a system. All participants had to find the rules and processes 'while running' their normal businesses.

Although attempts have been made to automate some of the registration process, but this turns out to be very difficult. The Product Information Management (PIM) system, which would have introduced standard forms of XML into the process grounded to a halt in 2011 [7].

At the moment, combinations of Microsoft Word files (version 2003; more modern versions 2007 and 2010 cannot be accepted [8]), and pdf-files are used. This practice makes it very difficult to control different versions, to check if modifications have been implemented, and to compare different files in different languages. Both pharmaceutical industry and regulatory authorities would like to develop a more practical and less time consuming approach because they agree that the current process is vulnerable.

\section{Alternative approach: performance based design}

The three assumptions in the previous section show the scope of the problem, and at the same time they provide a way forward. The approach that has been used since 1992 is to gradually change and modify the legislation and guidelines. Careful, step by step, progress was made. Unfortunately, the assumptions on which the legislation was based proved to be incorrect. Furthermore, the technology changed substantially, and best practice is very different from envisaged practice. Although it might be possible to 'upgrade current legislation' by small incremental changes, it is unlikely that these fundamental conflicts will disappear.

\subsection{Focus on actions in context}

Case studies [9] show that it is possible to develop prototypes that suit particular patients in a particular context if the first two assumptions are reversed. The starting points of these prototypes is that patients and medicines differ, and that writing, designing and testing are applied in an integral 'information development process'. Practical observations, user based design, performance based outcomes are all trained in education, developed in commercial practice, and investigated in experimental research.

This approach should be used as a basis for the development of information about medicines. The consequence of this is that 'performance based measures' need to be applied to studies that investigate the effectiveness of information in specific situations. This is 'best practice' in areas like usability, service design, and experience design. The transformation of this practice into legislation about medicines might be 
difficult, but is is likely to result in materials that 'really enable people to act appropriately'.

\subsection{Integrate digital and analogue modes.}

Most of the information about medicines that patients receive through the approved channels is still on paper. Combinations of analogue and digital methods have rarely been introduced until now. However, at the moment, most patients do have access to digital information, and it is time that personalized information about medicines is developed and becomes available in digital formats. This needs to be always in combination with information on paper and the relation between analogue and digital paper needs to be carefully considered and tested.

This practice is already common in other consumer domains. Phone bills, tax forms, and invoicing systems already deal with a combination of digital and analogue information. For the information about medicines, it is now possible to print personalized packaging, and personalized medicines regimen. 'Fridge door reminders', reminders as apps on smartphones, instructions on paper, and personalized e-mails are now all possible. Unfortunately, they are not developed further because the current regulatory framework does not allow for these artefacts.

Apart from the digital supply of information about medicines to patients, it is also necessary to develop electronic submission systems in a way that is similarly based on best practice and user-involvement. Most pharmaceutical industries use custom made digital document management systems. Unfortunately, there is hardly any standards across these systems, and the regulatory authorities should not be forced to familiarize themselves with all these differences. In this particular situation, standardization might be a real option.

\subsection{Test legislation, regulations, and guidelines}

Before any of the abovementioned changes is made, it is essential to conduct substantial testing. All stakeholders need to be involved to provide their criteria, and different types of tests are required. Unsufficiently tested materials will certainly waste substantial amount of time of most parties and might do more harm than good. The current regulations, templates, and guidelines quite clearly show the negative consequences.

\section{Conclusion}

In order to provide information about medicines that is evidence based, performance oriented and user centered it is necessary to develop a regulatory framework that supports the ambition to modify situations, and supports the control function of regulators. Four things need to be considered to achieve this: 
1. Medicines differ and patients differ. Standardisation is not an effective way to provide patients with relevant and appropriate information. It is essential to start from the perspective of patients and find patterns in their expectations and needs. Performance based information should be considered as a new assumption that underlies the next legislation.

2. Patients need reliable and independent information about their medicines that is guaranteed to be 'as correct as possible'. They expect a combination of digital and analogue information. The next legislation should allow for this.

3. For both regulators and industry, it becomes essential to see writing, designing, and testing as an integrated information development process. It seems necessary to provide regulators - who must check that information is correct and unbiassed - with advice and tools how they can really control the provision of reliable information. At the same time, the pharmaceutical industry needs to be supported to develop and submit information about medicines in standardised ways. It seems necessary to develop document management systems that support both.

4. None of these suggestions should be introduced without thorough testing in different contexts. Information about medicines needs to be tested with patients, and the regulatory framework needs to be tested with regulatory authorities and pharmaceutical industries.

Ultimately, we need to support and empower patients to take medicines correctly. The legal framework, and the economical considerations should become secondary. As long as legal arguments and profits prevail, patients will have to coop with visual information about medicines that does not really fulfil its potential.

\section{References}

1. EU Directive 2001/83/EU preliminary point 40.

2. EU Directive 2004/27/EU, article 63

3. EU Directive 2010/84/EU, amending article 59 of 2001/83.

4. Stickdorn, M. and Schneider, J. (2010). This is Service Design Thinking. Amsterdam, BIS Publishers.

5. EMA QRD-template version 9, (2013) (http://www.ema.europa.eu/docs/en_GB/document_library/Template_or_form/2009/10/WC 500004368.pdf

6. Readability guideline (2009) ( http://ec.europa.eu/health/files/eudralex/vol2/c/2009_01_12_readability_guideline_final_en.pdf)

7. http://pim.emea.europa.eu/

8 .

http://www.ema.europa.eu/ema/index.jsp?curl=pages/regulation/document_listing/document listing 000134.jsp

9. Sless, D. \&Shrensky, R.: Writing about medicines for people 3rd edition. Usability Guidelines for Consumer Medicine Information. Communication Research Institute (2006) 